Research Paper:

\title{
Antibacterial Effect of Zinc Oxide Nanoparticles on Standard Strains and Isolates of Pseudomonas Aeruginosa and Staphylococcus Aureus
}

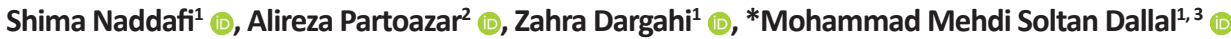

1. Department of Pathobiology, School of Public Health, Tehran University of Medical Sciences, Tehran, Iran.

2. Experimental Medicine Research Center, School of Medicine, Tehran University of Medical Sciences, Tehran, Iran

3. Food Microbiology Research Center, Tehran University of Medical Sciences, Tehran, Iran.

\begin{tabular}{|c|c|}
\hline $\begin{array}{l}\text { Use your device to scan } \\
\text { and read the article online }\end{array}$ & $\begin{array}{l}\text { Citation Naddafi S, Partoazar A, Dargahi Z, Soltan Dallal MM. [Antibacterial Effect of Zinc Oxide Nanoparticles on Standard Strains and } \\
\text { Isolates of Pseudomonas Aeruginosa and Staphylococcus Aureus Antibacterial Effect of Zinc Oxide Nanoparticles on Standard Strains } \\
\text { and Isolates of Pseudomonas Aeruginosa and Staphylococcus Aureus (Persian)]. Journal of Inflammatory Diseases. 2020; 24(3):234- } \\
\text { 245. https://doi.org/10.32598/JQUMS.24.3.4 }\end{array}$ \\
\hline 무굴 & doil'https://doi.org/10.32598/JQUMS.24.3.4 \\
\hline
\end{tabular}

Received: 15 Fev 2020 Accepted: 15 Jun 2020 Available Online: 01 Aug 2020

Keywords:

Zinc oxide nanoparticles, Pseudomonas aeruginosa, Staphylococcus aureus, Antibacterial activity

\section{A B STRACT}

Background Studies have shown that metal nanoparticles are highly active and exhibits remarkable bactericidal activity against a wide range of bacteria.

Objective The aim of this study was to examine the antibacterial activity of zinc oxide nanoparticles against standard strains of Pseudomonas aeruginosa and Staphylococcus aureus and their isolates in food products.

Methods This experimental study was conducted on the two pathogenic bacteria and their two standard strains. Zinc oxide nanoparticles were prepared from zeolite and their amount was determined using the XRF analyzer. Minimum Inhibitory Concentration (MIC) and Minimum Bactericidal Concentration (MBC) were measured using disk diffusion method .

Findings The MIC value of zinc oxide nanoparticles was $4 \mathrm{mg} / \mathrm{mL}$ for standard strain and isolate of pseudomonas aeruginosa and $2 \mathrm{mg} / \mathrm{mL}$ for standard strain and isolate of staphylococcus aureus. The MBC values for standard strain and isolate of pseudomonas aeruginosa were 16 and $8 \mathrm{mg} / \mathrm{mL}$, respectively, while for the standard strain and isolate of Staphylococcus aureus it was reported $8 \mathrm{mg} / \mathrm{mL}$.

Conclusion Staphylococcus aureus is more sensitive to zinc oxide nanoparticles that pseudomonas aeruginosa.

\section{Extended Abstract}

\section{Introduction}

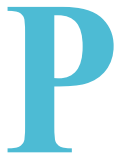

seudomonads are non-fermenting bacteria that are widely distributed in the environment. Pseudomonas aeruginosa (P. aeruginosa) is an important opportunistic pathogen that can quickly become resistant to drugs. It causes lung infections in people with cystic fibrosis and those need artificial respiration [1].
Staphylococci are among the first known human pathogens that colonize the skin and mucous membranes [3] One of the most important species of this pathogen is Staphylococcus aureus (S. aureus) which has become one of the major public health concerns due to its inherent ability and resistance to antimicrobial agents and drugs [4].

In recent years, metal oxides such as zinc oxide $(\mathrm{ZnO})$, have been considered as antimicrobial compounds. $\mathrm{ZnO}$ nanoparticles inhibit bacterial growth by producing hydrogen peroxide and penetrating the cell

\section{* Corresponding Author:}

Mehdi Soltan Dallal

Address: Food Microbiology Research Center, Tehran University of Medical Sciences, Tehran, Iran. Tel:

Tel: +98 (912) 1452646

E-Mail: msoltandallal@gmail.com 
wall and destroying the membrane; but its mechanism of action is still unclear [7-9].

In the study by Azam et al., the antibacterial effect of several nanoparticles on gram-positive and gram-negative bacteria was investigated. Their results showed that nanoparticles of $\mathrm{ZnO}$ had a better effect on both groups of bacteria [11]. In a study by Liu et al., ZnO nanoparticles could potentially be used as an effective antibacterial agent to protect the agriculture and food safety [13]. Due to a daily need for food in humans, any change in the food quality and quantity can have a significant impact on the community health. Removal of microbial contamination from food is important at any stages of food production, storage and supply [16]. The aim of this study was to compare the antibacterial effect of $\mathrm{ZnO}$ nanoparticles on standard strains of $\mathrm{P}$. aeruginosa and S. aureus isolated from food.

\section{Materials and Methods}

This experimental study was performed on two pathogenic and spoilage bacteria in meat and vegetable foods along with two standard strains of the same bacteria. In order to use the collected isolates, they were stored in a tryptic soy broth containing $15 \%$ of cultured glycerol at $-70^{\circ} \mathrm{C}$. The standard strains of P. aeruginosa (ATCC 27853) and S. aureus (ATCC 25923) used in this study were purchased from Zistroyesh Company in a freeze-dried form. In order to prepare the bacterial suspension for daily tests, McFarland suspension $\left(1-1.5 \times 10^{8} \mathrm{~mL}\right)$ was prepared. To ensure the correct turbidity of the McFarland suspension, its absorption was measured by a spectrophotometer at a wavelength range of $620 \mathrm{~nm}$ [17].

First, $100 \mathrm{~g}$ of zeolite with $70 \mathrm{~g}$ of zinc acetate was poured into $500-\mathrm{m}$ beaker and, then, $400 \mathrm{~mL}$ of deionized water was added to them. This beaker was then placed on a magnetic steering device. After 30 minutes, the beaker content was filtered using Whatman cellulose filter paper (Grade 40) and a white filter band (S\&S 589/2: 12-25 $\mu \mathrm{m}$, Germany) and washed by $500 \mathrm{~mL}$ of deionized water. Both filtered contents were then transferred to a glass plate and dried for 24 hours at room temperature. On the second day, the plate was incubated for 80 hours at $80^{\circ} \mathrm{C}$ and then was placed in a $120^{\circ} \mathrm{C}$ oven for 2 hours. The $400^{\circ} \mathrm{C}$ furnace was then used for calcinations of the obtained material for 2 hours [18]. Finally, to measure the amount of $\mathrm{ZnO}$, the XRF analyzer (PW 2404, Philips Co., Holland) was used available at the laboratory of Tarbiat Modares University.

The lowest concentration of nanoparticle suspension that did not show turbidity in the tube was determined as the Minimum Inhibitory Concentration (MIC) of nanoparticle growth. In tubes with no growth, Minimum Bactericidal Concentrations (MBC) was determined by performing a reculture on Müller-Hinton agar medium. These assessments were repeated three times [19]. All mediums used in this study were prepared from Merck Company in Germany. The antibacterial effect of $\mathrm{ZnO}$ nanoparticles on standard strains of P. aeruginosa and S. aureus isolated from food was evaluated by macrodilution method which includes the determination of MBC and MIC values and disk diffusion.

\section{Results}

Using the XRF analyzer, different percentages of elements in non-nano $\mathrm{ZnO}$ suspension and $\mathrm{ZnO}$ nanoparticle suspension were determined. The amount of $\mathrm{ZnO}$ nonnano $\mathrm{ZnO}$ suspension was obtained 8.358 and the amount of $\mathrm{ZnO}$ nanoparticle suspension was 25.149. The MIC of $\mathrm{ZnO}$ nanoparticle growth was reported $4 \mathrm{mg} / \mathrm{mL}$ for the standard strain and isolate of P. aeruginosa and $2 \mathrm{mg} / \mathrm{mL}$ for the standard strain and isolate of S. aureus. The MBC of $\mathrm{ZnO}$ nanoparticle suspension for the standard strain and $\mathrm{P}$. aeruginosa was obtained 16 and $8 \mathrm{mg} / \mathrm{mL}$, respectively, and for the standard strain and isolate of S. aureus was $8 \mathrm{mg} / \mathrm{mL}$

\section{Conclusion}

The results of this study showed that $\mathrm{ZnO}$ nanoparticle suspension had better antimicrobial effects on all bacteria compared to zeolite (non-nano $\mathrm{ZnO}$ ) . During this study, antibacterial activity increased with the increase in the concentration of the nanoparticle solution. Reddy et al. examined the antimicrobial effects of $\mathrm{ZnO}$ nanoparticles on $\mathrm{S}$. aureus and Escherichia coli and found that gram-positive $\mathrm{S}$. aureus was more sensitive to $\mathrm{ZnO}$ nanoparticles than gram-negative Escherichia coli, which is consistent with the results of our study [24]. Ramani et al. synthesized $\mathrm{ZnO}$ nanoparticles with different structures and examined its antibacterial properties on 4 strains of gram-positive bacteria and 4 strains of gram-negative bacteria, and observed that spherical $\mathrm{ZnO}$ nanoparticles had better antibacterial properties [24]. Seil et al. synthesized a composite of polyvinyl chloride and $\mathrm{ZnO}$ nanoparticles and studied its antibacterial effect on S. aureus and showed that $\mathrm{ZnO}$ improves the antibacterial properties of the study composite [25]. According to the results of the present study, it can be found that $\mathrm{S}$. aureus was more sensitive to $\mathrm{ZnO}$ nanoparticles than $\mathrm{P}$. aeruginosa.

It can be concluded that $\mathrm{ZnO}$ nanoparticles can be used in food packaging and storage as deterrents to pathogenic bacteria and food spoilage, leading to reduced consumption of raw materials and less waste in the packaging industry. The isolates of bacteria were more sensitive than the standard 
(Previous Title: The Journal of Qazvin University of Medical Sciences)

strains. Standard strains were clinical samples and become resistant over time due to the use of antibiotics.

\section{Ethical Considerations}

Compliance with ethical guidelines

This study obtained its ethical approval from the Ethics Committee of Tehran University of Medical Sciences (Code: IR.TUMS.VCR.REC.1397.484).

Funding

This study was extracted from the master thesis of first author, Department of Pathobiology, School of Public Health, Tehran University of Medical Sciences (Code: 39078).

Authors' contributions

Data collection, experiments, editing \& review: Shima Naddafi, Zahra Dargahi; Data analysis and interpretation: Mohammad Mehdi Soltan Dallal, Alireza Partoazar; Initial draft preparation: Shima Naddafi.

Conflicts of interest

The authors declare no conflicts of interest. 


\title{
مقايسه فعاليت ضدباكتريايى نانوذره اكسيد روى بر سويههاي استاندارد و جدايه بـودودوموناس أئروزينوزاو استافيلوكوك اورئوس ازئ مواد غذائيى
}

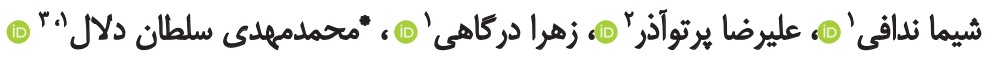

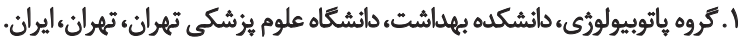

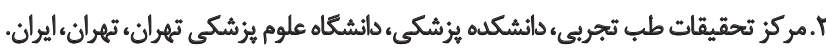

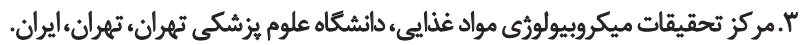

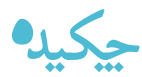

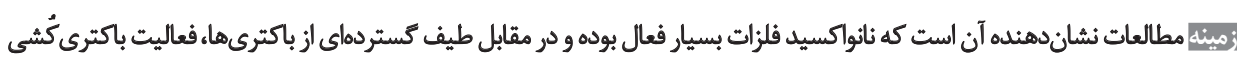
فوق العادالى نشان مي دهند.

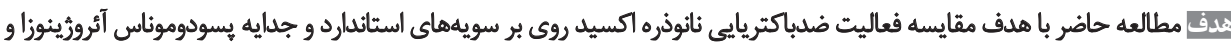

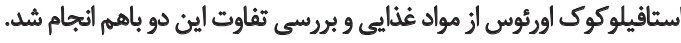

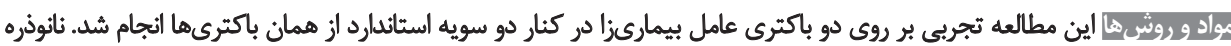

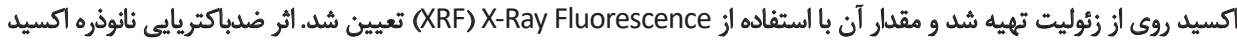

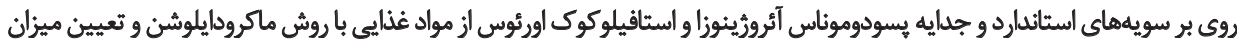
تو ويسك ديفيورن (MBC) Minimum Bactericidal Concentration , (MIC) Minimum Inhibitory Concentration

$$
\text { تعيين شد. }
$$

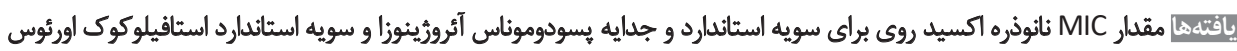

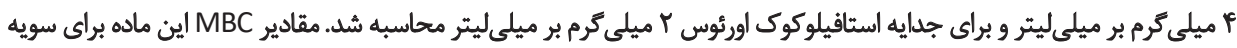

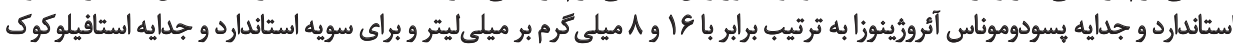

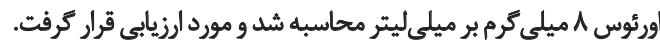

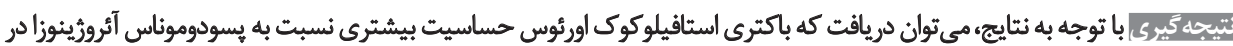
برابر نائوذرات اكسيد روى بارئ نارد.
تاريخ دريافت: 4 بهمن

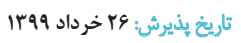
تاريخ انتشار: المرداد

تنفس مصنوعى استفاده مي كنند. اين باكترى عامل عفونتهاى

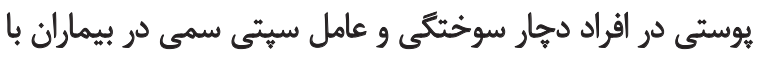

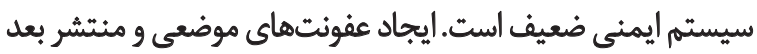

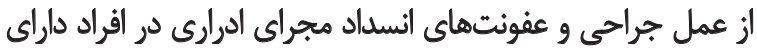

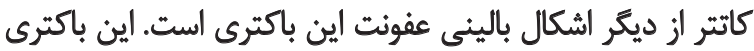

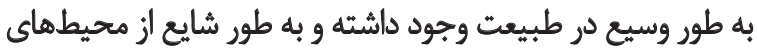
مرطوب بيمارستانى جدا شده است [1]

يسودوموناسها توانايى قابل توجهى در شكل گيرى بيوفيلم

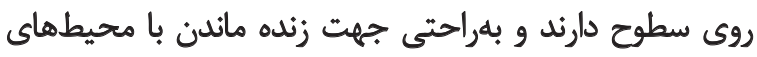

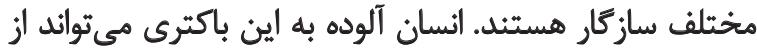

كليدواروهائ

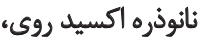

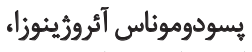

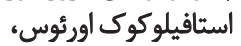
فعاليت
باكترىهاى يسودوموناس در خاك، آب، سطح كياهان و كلها،

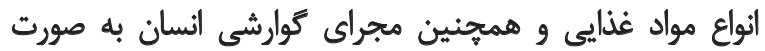

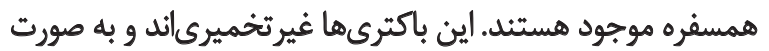

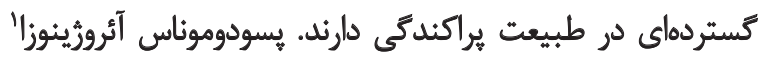

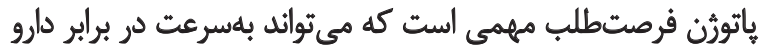

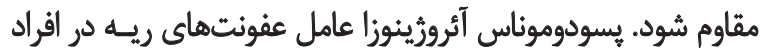

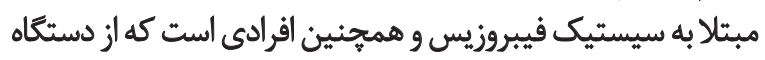

\section{Pseudomonas aeroginosa}

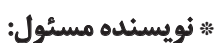

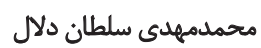

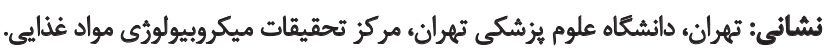
تلفئ رايانامه: msoltandallal@gmail.com 
بالقوه استفاده شود [11]. در مطالعه وانكَ" و همكاران اثرات

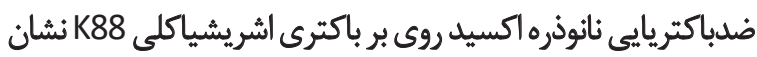

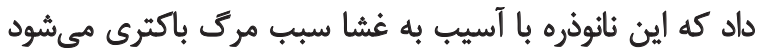

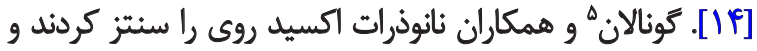

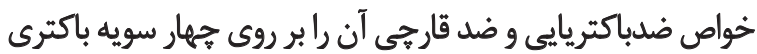

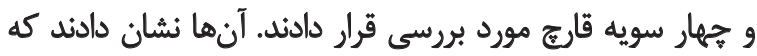

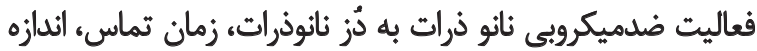
ذرات و روش سنتز بستكى دارد [1 10]. به دليل نياز روزمره انسان به مواد غذايى هركونه تغيير در برئ

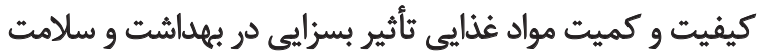

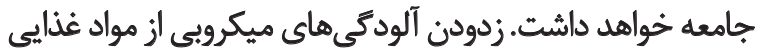

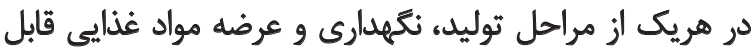

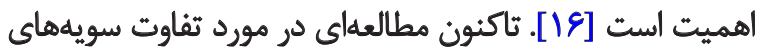

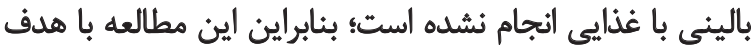

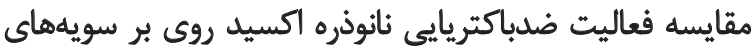

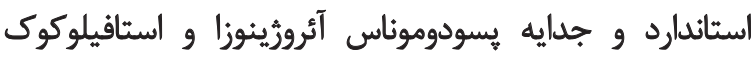
اورئوس از مواد غذايى و بررسى تفاوت اين دو باهم انجام كرفت.

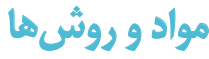

اين مطالعه از نوع تجربى روى دو باكترى بيمارىزا و عامل

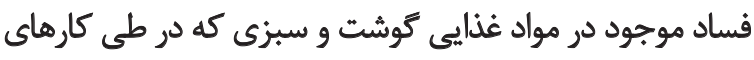

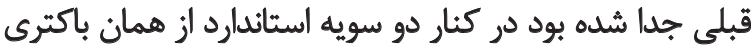

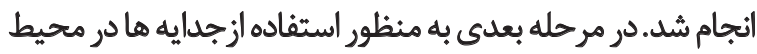

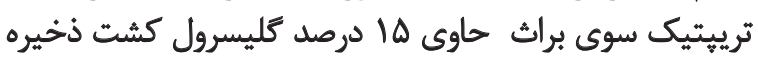

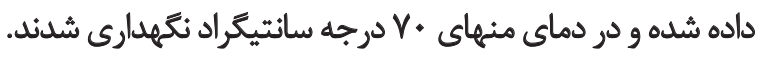
سويههاى استاندارد بِودوموناس آئروزينوزا ATCC 27853 و 250

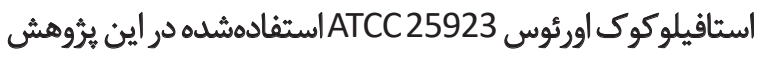

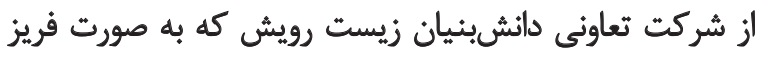

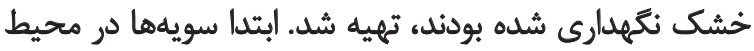

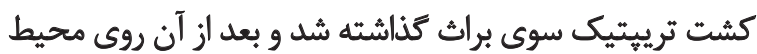

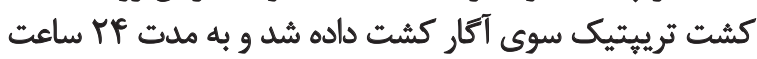

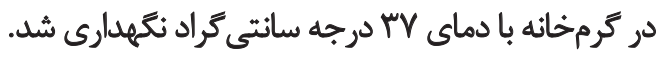
به منظور تهيه سوسيانسيون باكتريايى جهت انجام آزمايشات

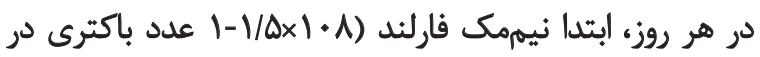

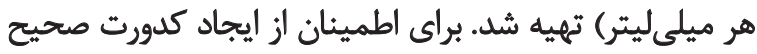

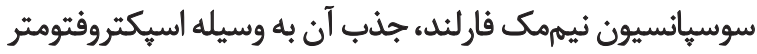

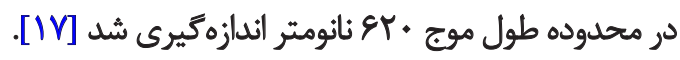
ابتدا زئوليت از نوع كلينويتيلوليت ؤسديم ــ بتاسيمى با خلوص

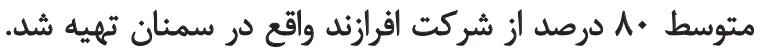

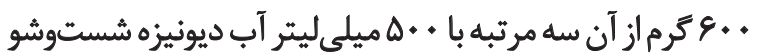

\section{Wang}

5. Gunalan

6. Clinoptilolite
طريق بزاق خود عامل انتفال بيمارى شود. به همين ترثيب

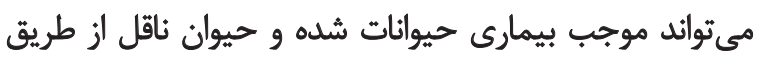

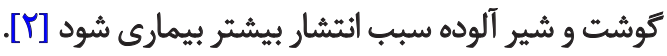

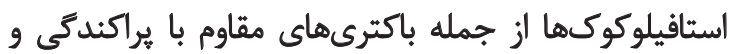

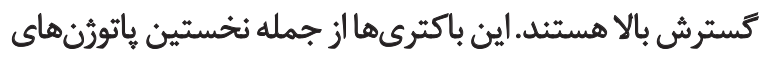

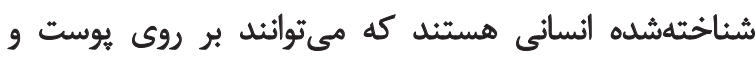

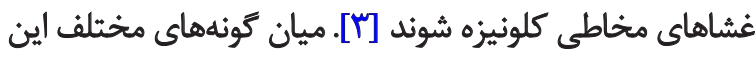

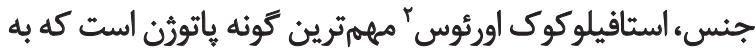

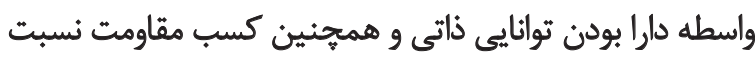

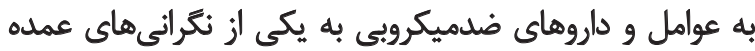

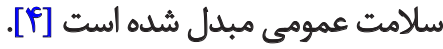

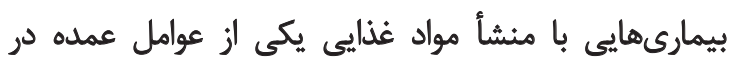

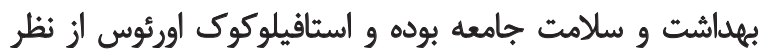

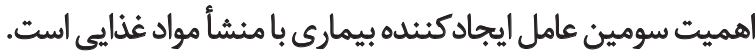

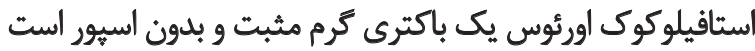

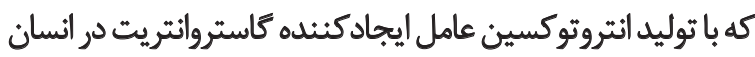

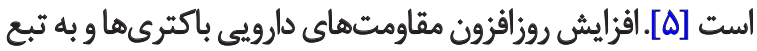

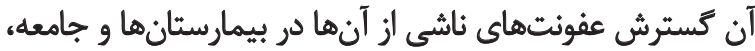

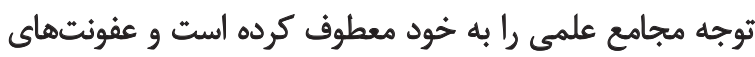

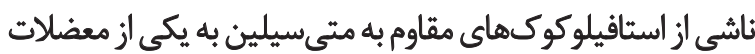

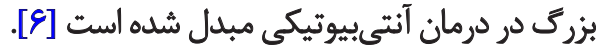
در سال هاى اخير، به اكسيدهاى فلزى، مانئد اكسيد روى به به

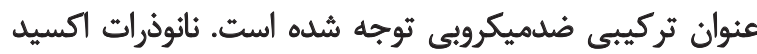

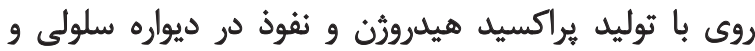

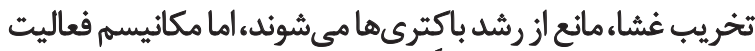

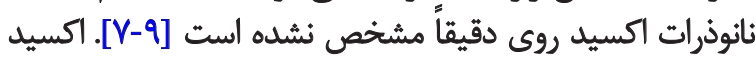

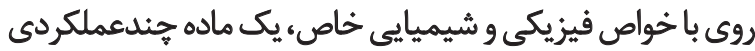

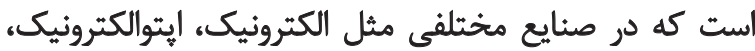

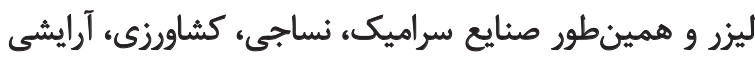

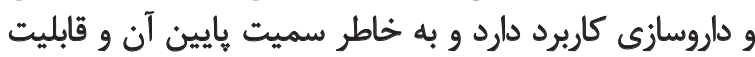

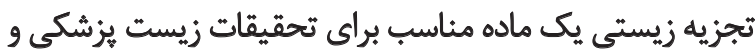

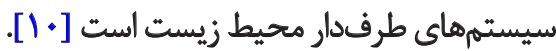

در تحقيق اعظهم و همكاران اثر ضدباكتريايى جند نائد نانواكسيد

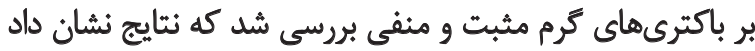

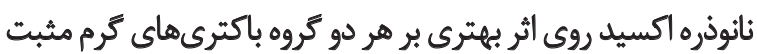

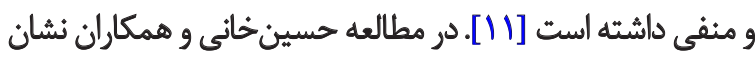

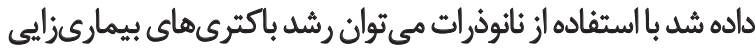

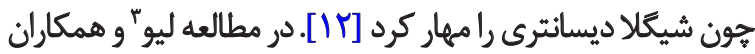

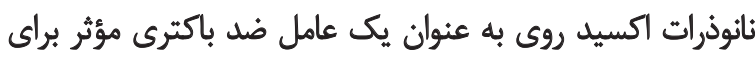

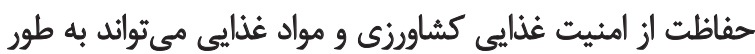

2. Staphylococcus aureus

3. Liu 


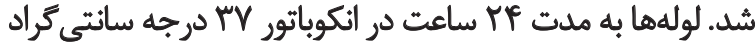

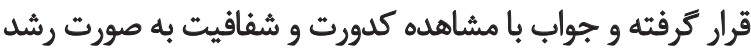

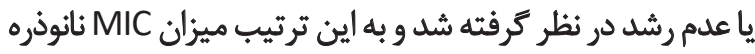

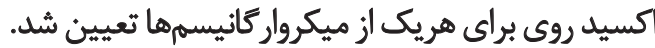

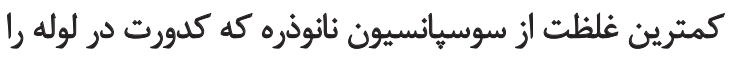

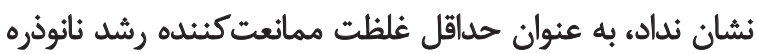

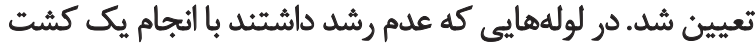

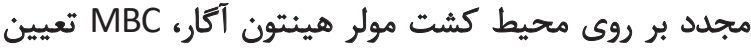

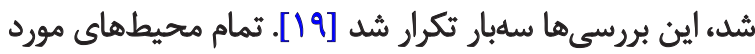
استفاده در اين مطالعه از شركت مرك آلمان تهيه شدر.

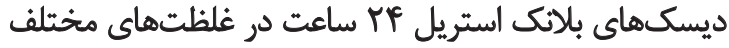

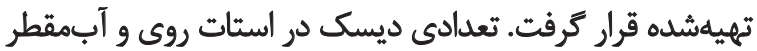

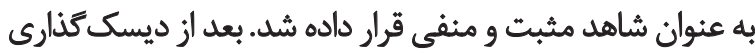

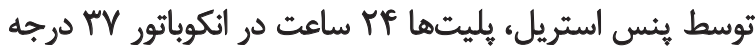

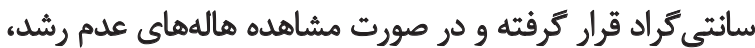

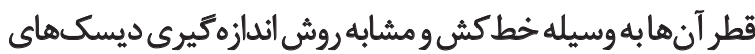

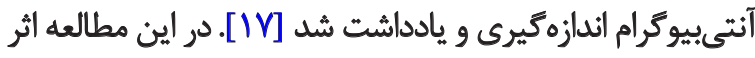

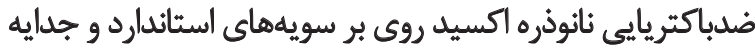

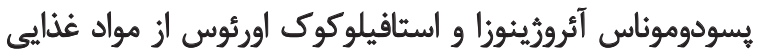

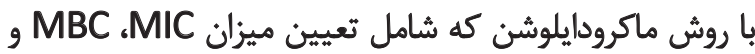
ديسك ديفيورن است، مورد ارزيابى قرار كرفت

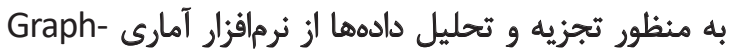

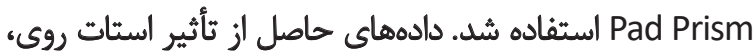
آبمقطر و غلظتهاى مختلف سوسيانسيون نانوذرات الكسيد

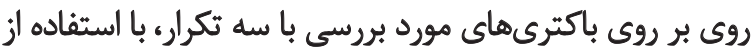

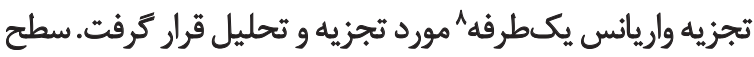
معنى دارى كمتر از هـ ٪ در نظر گرفتيه شد.

يأفتهن

با استفاده از دستكاه XRF درصدهاي مختلف عناصر موجود در

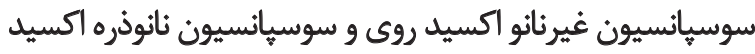
روى تعيين شد كه مقدار اكسيد روى در سوسيانيانسيون غيرنانيانو

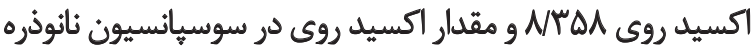

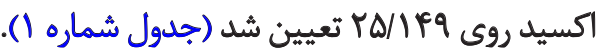

مقادير حداقل غلظت بازدارنده رشد سوسيانسيون نانوذره

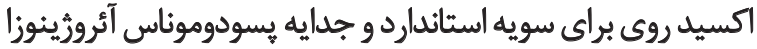

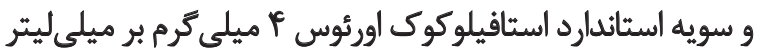

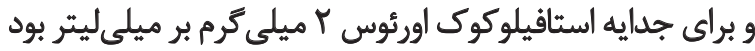

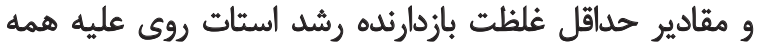

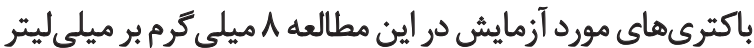
و سوسيانسيون كاميوزيت غير نانو اكسيد روى و زئوليت فائد

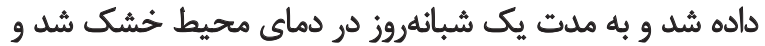

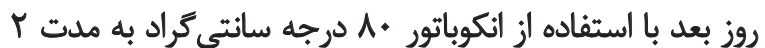

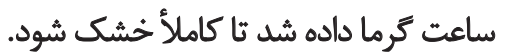

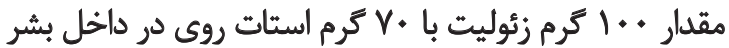

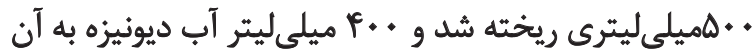

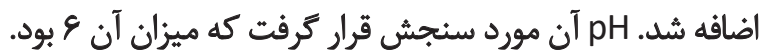

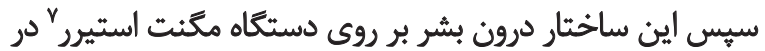

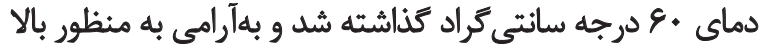

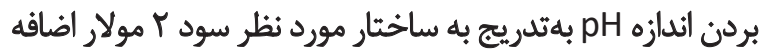

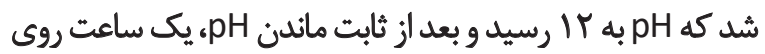

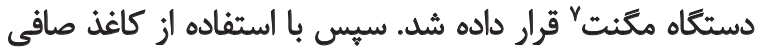

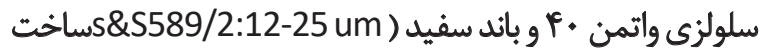
آلمان) محتواى بشر فيلتر شد.

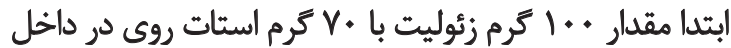

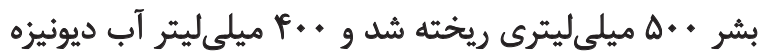

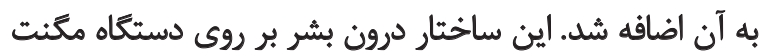

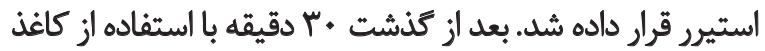

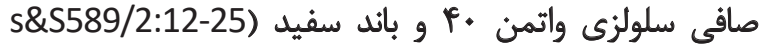

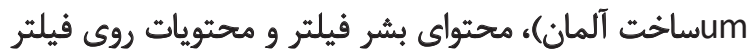

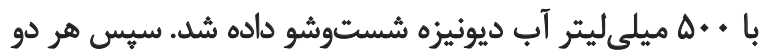

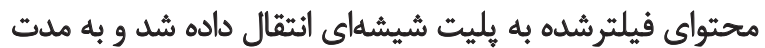

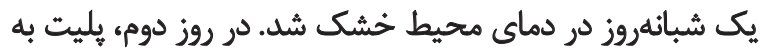

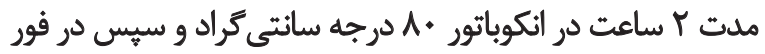

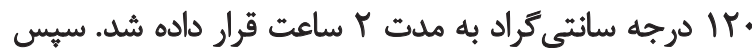

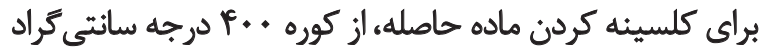

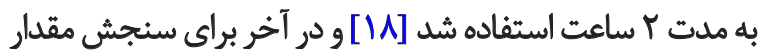
XRF (Model: PW 2404, Com- زينك اكسايد، از دستخاي موجود در آزمايشعاه دانشعاه تربيت مدرس استفاده شد.

از هر جهار نمونه، نانوذره اكسيد روى و كاميوزيت غيرنانو ئون

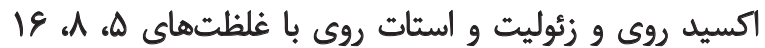

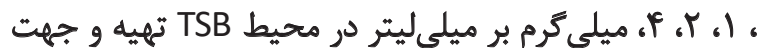

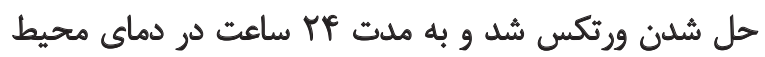

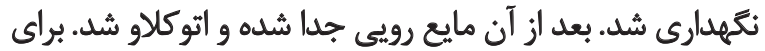

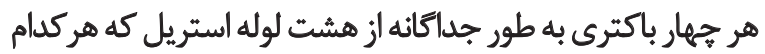

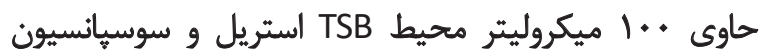

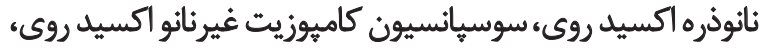

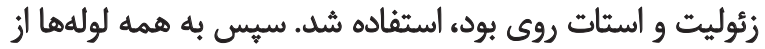

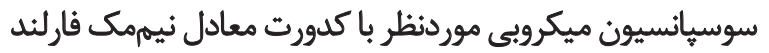

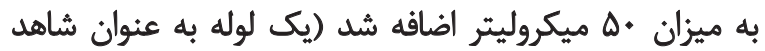

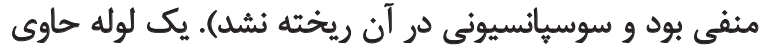
سوسيانسيون ميكروبى فاقد نانوذره به عنوان شاهد مثبت نشيت استفاده

7. Magnet 
A

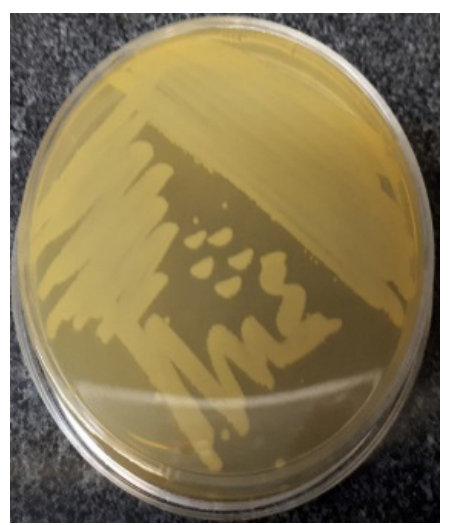

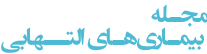

B

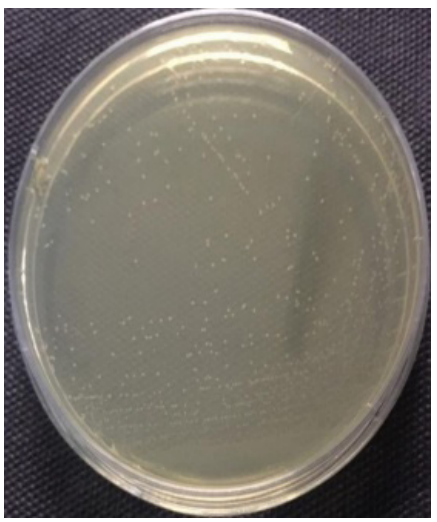

C

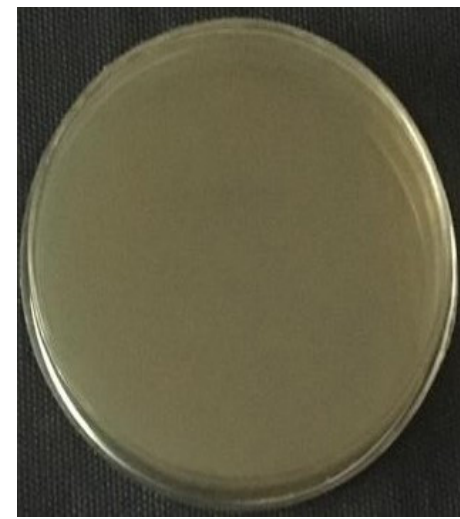

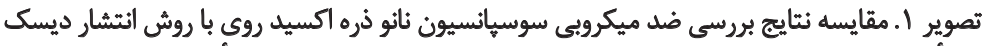

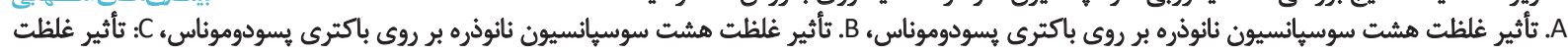

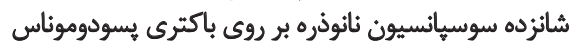

باكترىها اثرات ضلميكروبى بهترى نسبت به سوسيانسيون

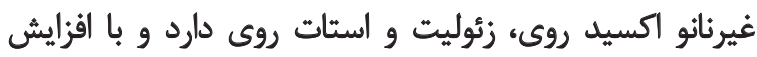

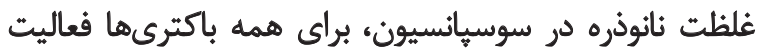
ضدباكتريايي افزّايش مي نيابد.

سوسيانسيون نانوذرهاكسيد روى بر باكترى يسودوموناس آئروزينوزا

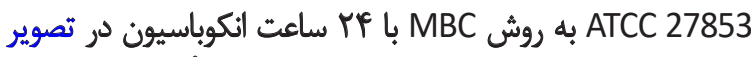

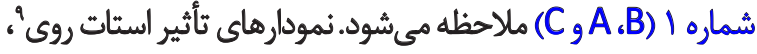

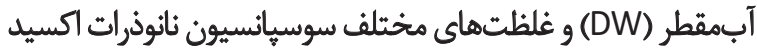

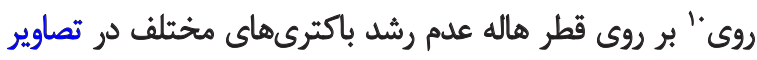

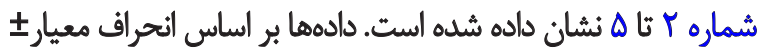
ميانكين با سه تكرار براي همه كروهها بروسي شاده شده است.

9. $\mathrm{Zn}$ ac 10. ZnO Nano
حداقل غلظت بازدارنده رشد، عليه تمامى باكترىهاى مورد آزمايش در اين مطالعه بودند.

مقادير حداقل غلظت كشندگى سوسيانسيون نانوذره اكسيد روى

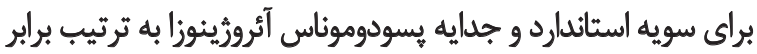

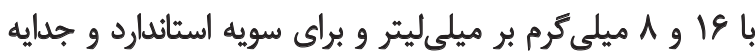

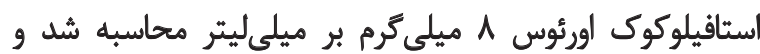

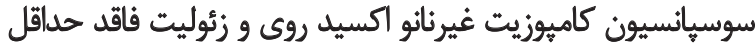

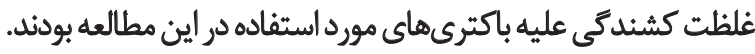

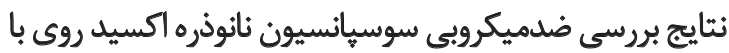

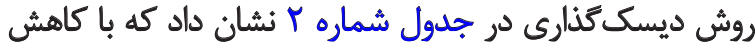

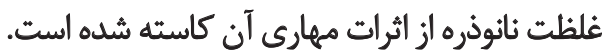

نتايج نشان مي دهد سوسيانسيون نائوذره اكسيد روى بر همه

جدول (. مقايسه درصدهاي مختلف عناصر موجود در سوسيائسيون غيرنائو اكسيد روى و سوسيانسيون نائوذره اكسيد روى

\begin{tabular}{|c|c|c|c|c|c|c|c|c|c|c|c|c|}
\hline TiO2 & $\mathrm{CaO}$ & K2O & $\mathrm{Cl}$ & SO3 & P2O5 & SiO2 & & Al2O3 & MgO & L.O.I. & عناصر & \\
\hline ./NF & rise & I/MTF & .1 .5 & . /NAT & $.1 \cdot m$ & ED/AIq & & $N Q \cdot D$ &.$/ D \& A$ & V/IT & درصد & سوسيانسيون غيرنائو \\
\hline.$/ M V$ & $P / M$ & $1 / . r 9$ & $1 .+9$ & $. N+r$ & $.1 . r$ & $\Delta . / M T H$ & & V/ETT &.$/ 4 V A$ & V. & درصد & سوسيانسيون ناثوذره \\
\hline $\mathrm{Pb}$ & $\mathrm{Ba}$ & $\mathrm{Zr}$ & $\mathrm{Sr}$ & $\mathbf{R b}$ & $\mathrm{ZnO}$ & $\mathrm{Cu}$ & $\mathrm{Ni}$ & Co & $\mathrm{Fe} 2 \mathrm{O} 3$ & MnO & عناصر & \\
\hline ol.r &.$/ M T$ & $1 . r 8$ &.$/ M$ & .1 .04 & NTM & $.1 \% \Delta$ & $.1 \cdot r^{2}$ & $.1 . r$ & I/T91 & /.AT & درصد & 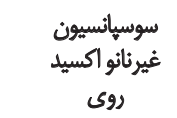 \\
\hline I. MT & - Mar & 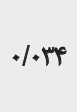 & . & 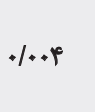 & $r \Delta / / P q$ & & .10 .9 & & I/MaP & $1 .+9$ & خرصد & ناثوذره اكسيديد \\
\hline
\end{tabular}

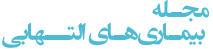


جدول r. تأثير غلظتهاى مختلف نانوذره اكسيد روى بر روى باكترىهاى بِسودوموناس آئروزينوزا و استافيلوكوك اورئوس، بر اساس قطر هاله عدم رشد

باكترى

\begin{tabular}{|c|c|c|c|c|c|c|c|c|}
\hline - & 1. & ir & 11 & 1. & 9 & - & - & ATCC 27853 يسودوموناس آتروزينوزا \\
\hline • & 1. & 10 & ir & 11 & 1. & . & • & جدايه يسودوموناس آثروزينوزا \\
\hline - & 1. & ir & if & $\pi$ & 11 & - & $\cdot$ & ATCC استافيلوكوى اورئوس 25923 \\
\hline - & 1. & 19 & 18 & ir & ir & - & $\cdot$ & جدايه استافيلوكوى اورئوس \\
\hline
\end{tabular}

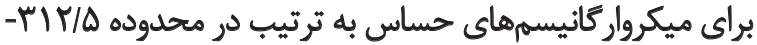

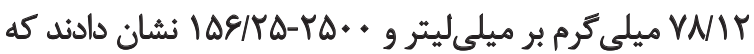

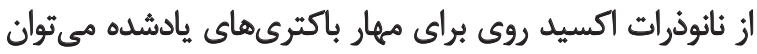

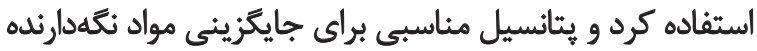

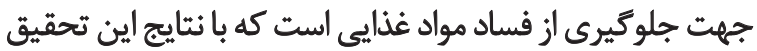

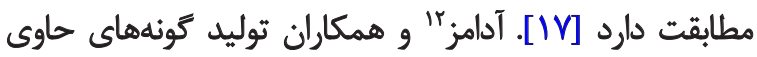

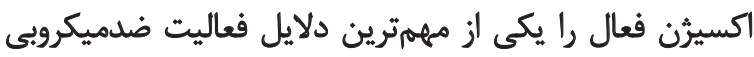

نانوذرات اكسيد روى ذكر كردهاند [19].

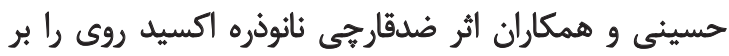

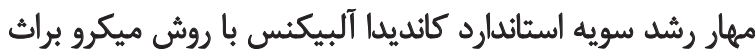

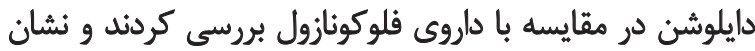

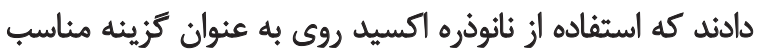

12. Adams

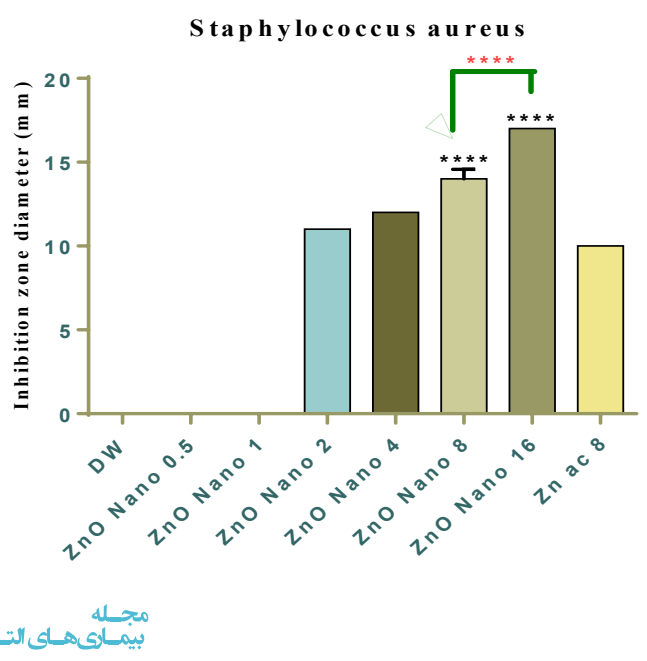

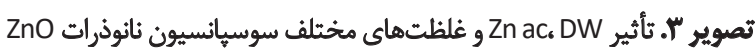

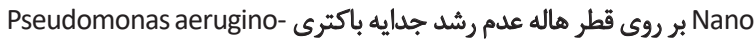

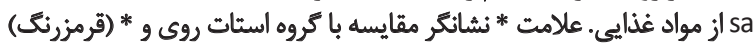

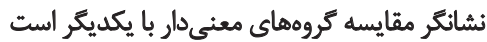
. $\left.\left(P \leq+1 \cdot \Delta^{* * * * *} \text { ، } P \leq+1+\cdot\right)^{*}\right)$

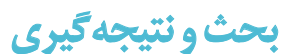

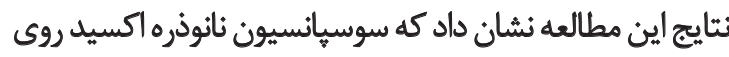

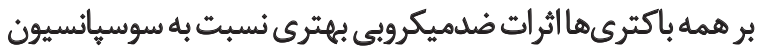

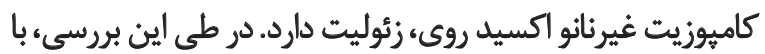

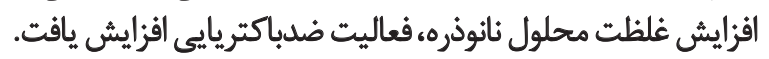

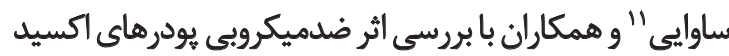

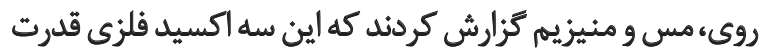

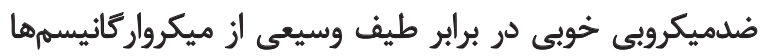

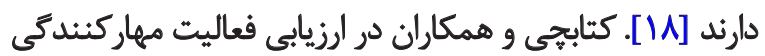

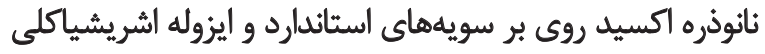

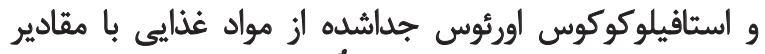

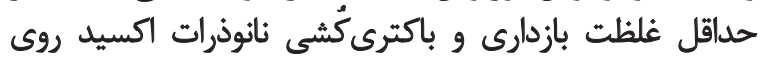

11. Sawai

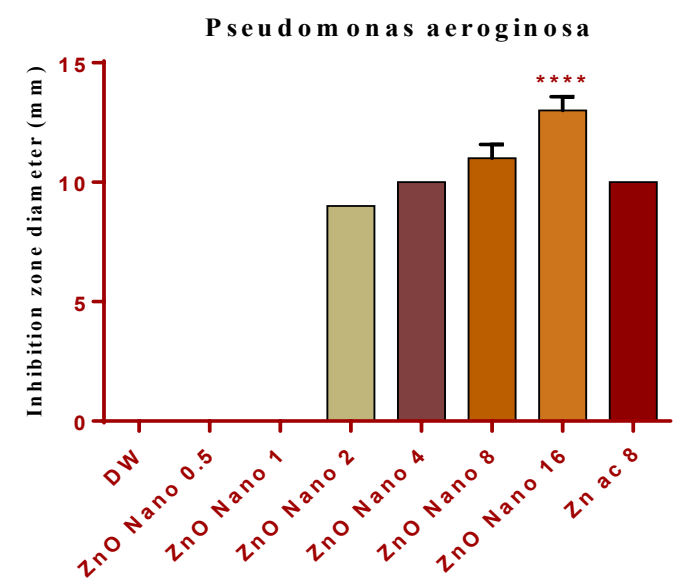

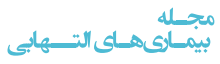

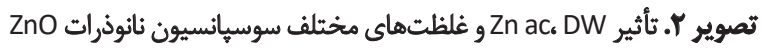

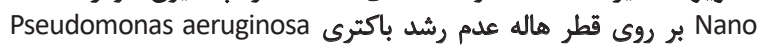

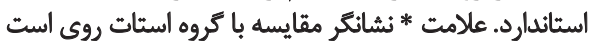
. (P $\leq 0 / 0001^{* * * * *}$ ) 
Staphylococcus aureus (Isolated)

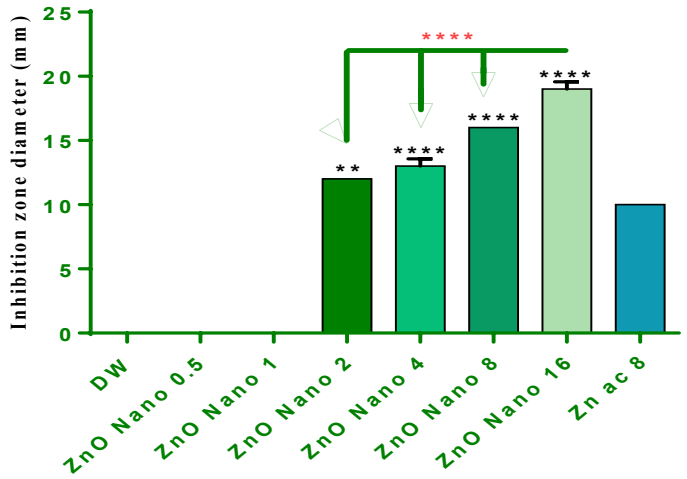

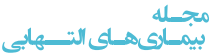

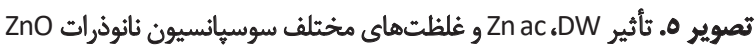

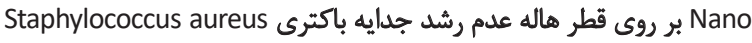

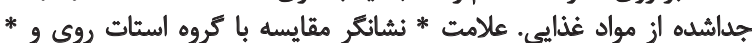

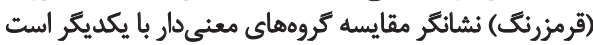

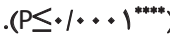

نانوذرات اكسيد روى را سنتز كردند و خواص ضدباكتريايى آن

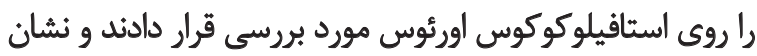
دادند كه اكسيد روى باعث بهببود عملكرد خاصني

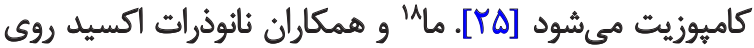

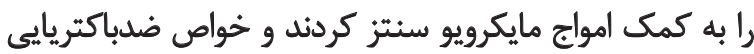

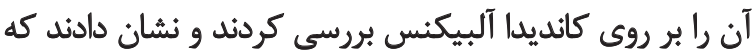

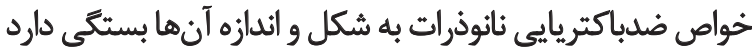

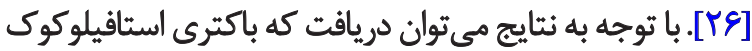

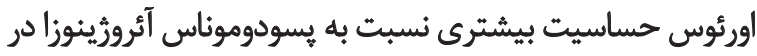

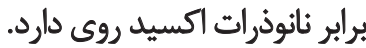

بر اساس نتايج اين يُؤوهش، نانوذرات اكسيد روى مي توانئد

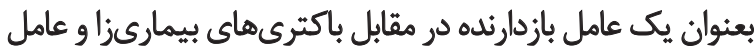

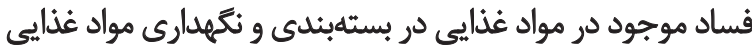

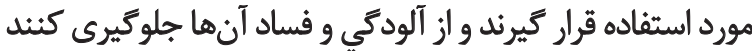

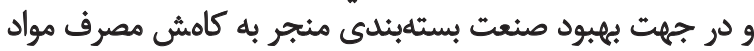

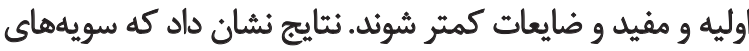

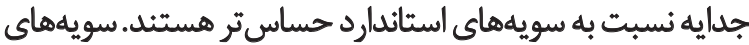

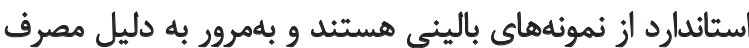

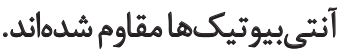

مالاحظاث أخلاقي

$$
\text { يبيروى از اصول اخلاق يؤهش }
$$

اين بروهش در كميته اخلاق دانشكاه علوميزشكى تهران باكُد

18. $\mathrm{Ma}$

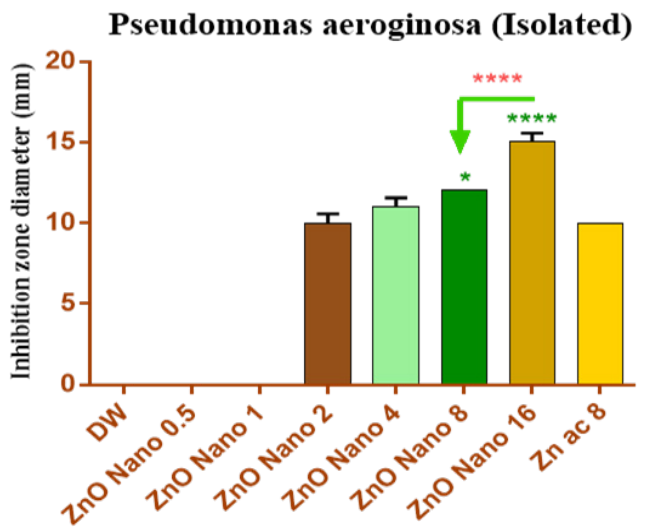

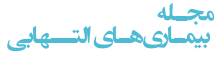

تصوير ع. ثأثير ZnO ac ، و غلظتهاي مختلف سوسيانسيون نانوذرات

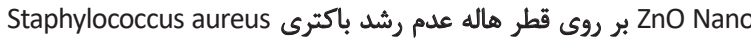

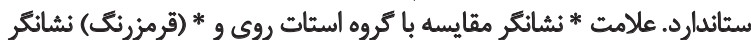

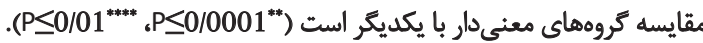

براى حذف كانديدا آلبيكنس در حيطه بزشكى، بلهويره در ارتباط با بائ

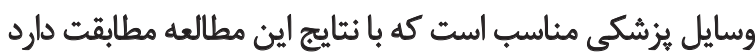

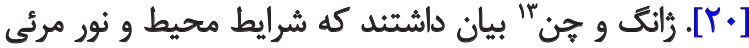

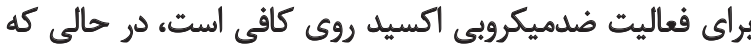

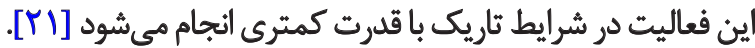
سينها" و همكاران در بررسى اثرات ضدميكروبى نانوذرات اكسيد

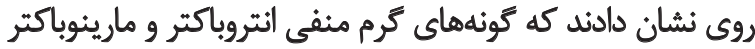

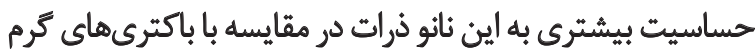

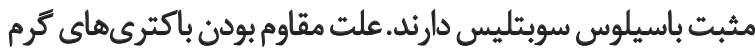

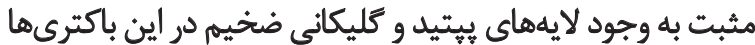

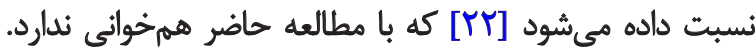

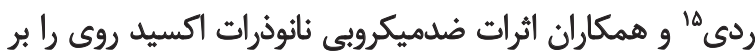

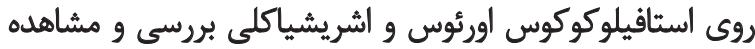

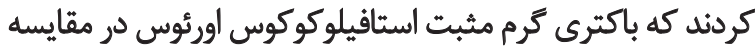

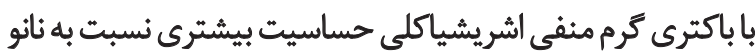
ذرات اكسيد روى دارد كه با نثايج اين تحقيق مطابقت دارد [بكائ. رامانى" و همكاران نانوذرات اكسيد روى را با ساختارهاى

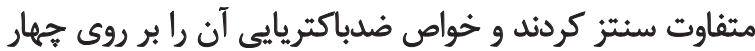

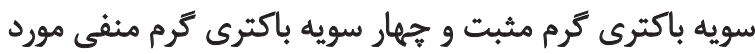

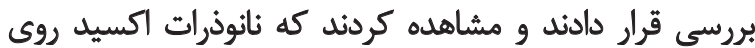

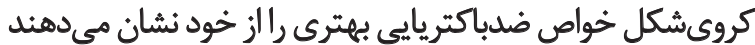

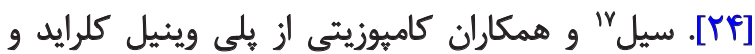

13. Zhang \& Chen

14. Sinha

15. Reddy

16. Ramani

17. Seil 


$$
\text { مامى مالى }
$$

اين مقاله مستخرج از ياياننامه كارشناسي ارشد نويسنده اول

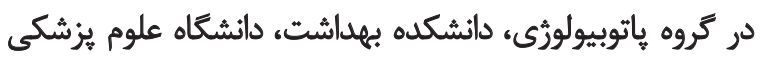

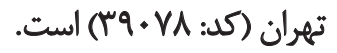

$$
\text { مشاركت نويسندكَان }
$$

جمعآورى دادهها، انجام آزمايشات و نتيجهيرى، اصلاح

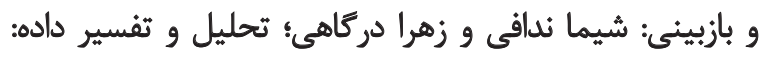

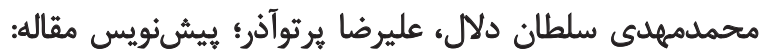
شيماندافي. محمديد.

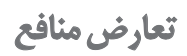

بنابر اظهار نويسندكان اين مقاله تعارض منافع ندارد. 


\section{References}

[1] Raposo A, Pérez E, de Faria CT, Ferrús MA, Carrascosa C. Food Spoilage by Pseudomonas spp. An Overview. In: Singh OV, editor. Foodborne pathogens and antibiotic resistance. $1^{\text {th }}$ edition. New Jersey: John Wiley \& Sons, Inc; 2017 [DOI:10.1002/9781119139188.ch3]

[2] Japooni A, Alborzi A, Orafa F, Rasouli M, Farshad S. Distiribution patterns of methicillin resistance genes (mecA) in staphylococcus aureus Isolated from clinical speciments. Iran Biomed J. 2004; 8(4):173-8. http://ibj.pasteur.ac.ir/article-1-489-en.html

[3] Rahimi F, Bouzari M, Katouli M, Pourshafie MR. Antibiotic resistance pattern of methicillin resistant and methicillin sensitive Staphylococcus aureus Isolates in Tehran, Iran. Jundishapur J Microbiol. 2013; 6(2):144-9. [DOI:10.5812/jjm.4896]

[4] Boerema JA, Clemence R, Brightwell G. Evaluation of molecular methods to determine enterotoxigenic status and molecular genotype of bovine, ovine, human and food isolates of staphylococcus aureus. Int j Food Microbiol. 2006; 107(2):192-201. [DOI:10.1016/j.ijfoodmicro.2005.07.008] [PMID]

[5] Pereira V, Lopes C, Castro A, Silva J, Gibbs P, Teixeira P. characterization for enterotoxin production, Virulence factors, and antibiotic susceptibility of staphylococcus aureus isolates from various foods in Portugal. Food Microbiol 2009; 26(3):278-82. [DOI:10.1016/j.fm.2008.12.008] [PMID]

[6] Emami-Karvani Z, Chehrazi P. Antibacterial activity of ZnO nanoparticle on gram positive and gram-negative bacteria. Afr J Microbiol Res. 2011; 5(12):1368-73. [DOI:10.5897/AJMR10.159]

[7] Dutta RK, Nenavathu BP, Gangishetty MK, Reddy AVR. Studies on antibacterial activity of ZnO nanoparticles by ROS induced lipid peroxidation. Colloids Surf B Biointerfaces, 2012; 94:143-50. [DOI:10.1016/j.colsurfb.2012.01.046] [PMID]

[8] Xie Y, He Y, Irwin PL, Jin T, Shi X. Antibacterial activity and mechanism of action of zinc oxide nanoparticles. J of Appl Environ Microbiol. 2011; 77(7):2325-31. [DOI:10.1128/AEM.02149-10] [PMID] [PMCID]

[9] Ravikumar S, Gokulakrishnan R, Boomi P. In vitro antibacterial activity of the metal oxide nanoparticles against urinary tract infectious bacterial pathogens. Asian Pac J Trop Dis. 2012; 2(2):85-9. [DOI:10.1016/S2222-1808(12)60022-X]

[10] Kołodziejczak-Radzimska A, Jesionowski T. Zinc oxide-from synthesis to application: A review. Materials (Basel). 2014; 7(4):283381. [DOI:10.3390/ma7042833] [PMID] [PMCID]

[11] Azam A, Ahmed AS, Oves M, Khan MS, Habib SS, Memic A. Antimicrobial activity of metal oxide nanoparticles against Grampositive and Gram-negative bacteria: A comparative study. Int J Nanomedicine. 2012; 7:6003-9. [DOI:10.2147/IJN.S35347] [PMID] [PMCID]

[12] Hosseinkhani P, Zand AM, Imani S, Rezayi M, Rezaei Zarchi S. Determining the antibacterial effect of $\mathrm{ZnO}$ nanoparticle against the pathogenic bacterium, Shigella dysenteriae (type 1). Int J Nano Dim. 2011; 1(4):279-85. [DOI:10.7508/IJND.2010.04.006]

[13] Liu Y, He L, Mustapha A, Li H, Hu Z, Lin M. Antibacterial activities of zinc oxide nanoparticles against Escherichia coli 0157: H7. J App Microbial. 2009; 107(4):1193-201. [DOI:10.1111/j.13652672.2009.04303.x] [PMID]
[14] Wang C, Liu L-L, Zhang A-T, Xie P, Lu J-J, Zou X-T. Antibacterial effects of zinc oxide nanoparticles on Escherichia coli K 88. Afr J Biotechnol. 2012; 11(44):10248-54. [DOI:10.5897/AJB11.3703]

[15] Gunalan S, Sivaraj R, Rajendran V. Green synthesized ZnO nanoparticles against bacterial and fungal pathogens. Pro Nat SciMater. 2012; 22(6):695-702. [DOI:10.1016/j.pnsc.2012.11.015]

[16] Alswat AA, Ahmad MB, Saleh TA, Hussein MZB, Ibrahim NA. Effect of zinc oxide amounts on the properties and antibacterial activities of zeolite/zinc oxide nanocomposite. Mater Sci Eng C Mater Biol Appl. 2016; 68:505-11. [DOI:10.1016/j.msec.2016.06.028] [PMID]

[17] Ketabchi M, lessazadeh KH, Massiha A. Evaluate the inhibitory activity of ZnO nanoparticles against standard strains and isolates of Staphylococcus aureus and Escherichia coli isolated from food samples. J Food Microbiol. 2017; 4(1):6374. [In Persian] http://jfm.iaushk.ac.ir/article_654468_8b33 49bceb43296f4d28999b4a3fe05c.pdf

[18] Sawai J, Yoshikawa T. Quantitative evaluation of antifungal ac tivity of metallic oxide powders ( $\mathrm{MgO}, \mathrm{CaO}$ and $\mathrm{ZnO}$ ) by an indirect conductimetric assay. J Appl Microbiol. 2004; 96(4):803-9. [DOI:10.1111/j.1365-2672.2004.02234.x] [PMID]

[19] Adams LK, Lyon DY, Alvarez PJJ. Comparative ecotoxicity of nanoscale TiO2, SiO2, and ZnO water suspensions. Watter Res. 2006; 40(19):3527-32. [DOI:10.1016/j.watres.2006.08.004] [PMID]

[20] Hosseini SS, Joshagani HR, Eskandari M. Colorimetric MTT assessment of antifungal activity of $\mathrm{ZnO}$ nanowires against candida dubliensis bioflm. Jundishapur J Health Sci. 2013; 12(1):69-80. [In Persian] http://pdfarchive.ir/pack-5/Do_52513928205.pdf

[21] Zhang $\mathrm{H}$, Chen G. Potent antibacterial activities of $\mathrm{Ag} / \mathrm{TiO} 2 \mathrm{na}$ nocomposite powders synthesized by a one-pot sol-gel method. Environ Sci Technol. 2009; 43(8):2905-10. [DOI:10.1021/ es803450f] [PMID]

[22] Sinha R, Karan R, Sinha A, Khare SK. Interaction and nanotoxic effect of $\mathrm{ZnO}$ and $\mathrm{Ag}$ nanoparticles on mesophilic and halophilic bacterial cells. Bioresour Technol. 2011; 102(2):1516-20. [DOI:10.1016/j.biortech.2010.07.117] [PMID]

[23] Reddy KM, Feris K, Bell J, Wingett DG, Hanley C, Punnoose A. Selective toxicity of zinc oxide nanoparticles to prokaryotic and eukaryotic systems. Appl Phys Lett. 2007; 90(21):1-3. [DOI:10.1063/1.2742324] [PMID] [PMCID]

[24] Ramani M, Ponnusamy S, Muthamizhchelvan C. From zinc oxide nanoparticles to microflowers: A study of growth kinetics and biocidal activity. Mater Sci Eng. 2012; 32(8):2381-9. [DOI:10.1016/j. msec.2012.07.011]

[25] Seil JT, Webster TJ. Reduced Staphylococcus aureus proliferation and biofilm formation on zinc oxide, nanoparticle PVC composite surfaces. Acta Biomater. 2011; 7(6):2579-84. [DOI:10.1016/j.actbio.2011.03.018] [PMID]

[26] Ma J, Liu J, Bao Y, Zhu Z, Wang X, Zhang J. Synthesis of largescale uniform mulberry-like $\mathrm{ZnO}$ particles with microwave hydrothermal method and its antibacterial property. Ceram Int. 2013; 39(3):2803-10. [DOI:10.1016/j.ceramint.2012.09.049] 
This Page Intentionally Left Blank 